\title{
Oesophageal propulsive force and its relation to manometric pressure
}

\author{
C O H Russell, N Bright, G Buthpitiya, L Alexander, C Walton, G Whelan
}

\begin{abstract}
A fixed volume capsule incorporating a force transducer and a side hole for manometric measurements was constructed and calibrated. Simultaneous measurements of the propulsive (aboral) force and the manometric pressure (intraluminal pressure) were made at 5,10 , and $15 \mathrm{~cm}$ above the lower oesophageal sphincter and in response to dry and wet $(5,10$, and $15 \mathrm{ml}$ ) swallows. The propulsive force and manometric pressure waves had a simultaneous onset and were of similar duration. Peak values of propulsive force for wet swallows increased significantly as measurements were made progressively more distally within the oesophagus and were greatest in the distal oesophagus. The association between manometric pressure and propulsive force is not strong $(r=0.61)$ suggesting that intraluminal pressure is a poor predictor of propulsive force and hence an unreliable measure of oesophageal 'function'.
\end{abstract}

Oesophageal peristalsis acts in concert with the pharynx to propel swallowed material into the stomach. Currently, perfused side hole manometry is used to assess peristaltic function. The peak manometric pressure reflects the maximum intraluminal pressure developed in response to the peristaltic wave. Our own manometric studies ${ }^{1}$ in asymptomatic normal volunteers confirmed the existence of a wide range of normal values of peak amplitude, duration, and velocity. Analysis of variance indicated this variation was mainly among individuals implying that normal oesophageal transport can occur within a wide range of values of peristaltic amplitude and that peristaltic amplitude in the range seen in normal volunteers may have little predictive value regarding the efficiency of oesophageal function. The vital force vector that results from peristaltic action must work in an aboral direction. This aboral force has been ascertained in the past, ${ }^{23}$ using 'linear strain gauge' assemblies to measure the distracting force acting on a detachable sphere. One study ${ }^{2}$ showed that the relationship between maximal peristaltic pressure and force, although positive, was weak and the other ${ }^{3}$ concluded that manometric pressure 'was a good index of peristaltic force'. Both these studies had methodological limitations that may have affected the results. In Pope's study manometric pressure was measured adjacent to the sphere by continuous perfusion side hole $(\mathrm{CPSH})$ manometry with a syringe pump. Schoen $e t a l^{3}$ in a similar study also used syringe pumps for continuous perfusion side hole manometry, but with high infusion rates $(6 \mathrm{ml} / \mathrm{min})$ in an attempt to avoid undermeasurement of manometric pressure because of compliance of syringe pumps. ${ }^{+}$ This latter study omits to describe the character or the volume of the bolus used to initiate peristalsis. Neither study performed a calibration of the strain gauge at body temperature (vide infra) although Schoen et al do state that 'temperature did not affect calibration'. These methodological limitations were believed to be of significant importance to reevaluate the situation.

The aims of this present study were (a) to develop a linear strain gauge system that would permit a detailed examination of the relationship between measures of peristalsis obtained by a low compliance manometric system and the aboral propulsive force generated simultaneously at the same site and (b) to study the effect of bolus volume on this propulsive force.

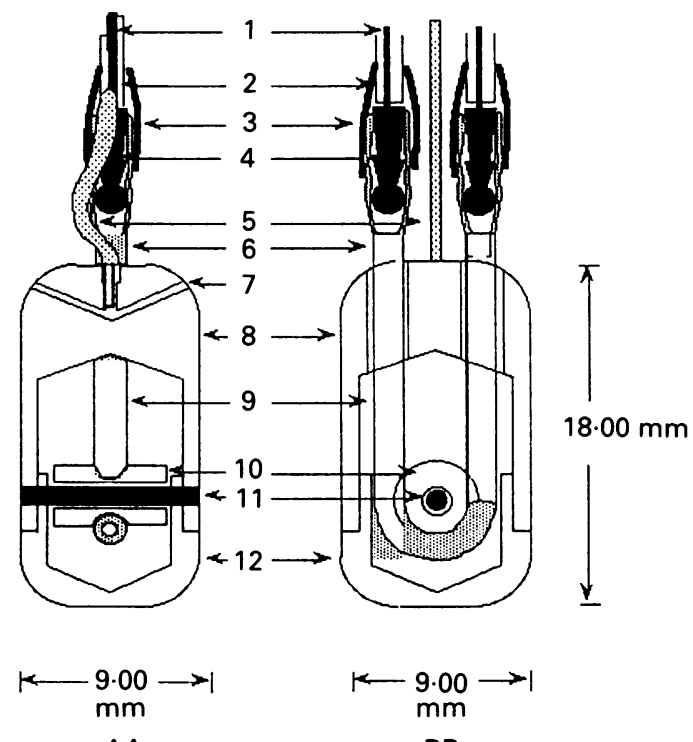

AA

BB

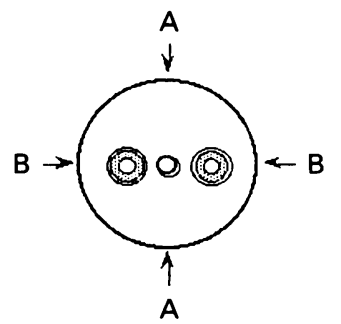

Figure 1: Diagram of linear transducer/catheter assembly. The various parts are: $1:$ insulated copper wire to impedance monitor, 2: clear vinyl manometry catheter assembly, 3: hea shrink tubing, 4: manometry tubing to port in capsule, 5: electrode, 6: silastic tubing, 7: capsule manometry port, 8: upper capsule body, 9: silastic loop, 10: roller, 11: retaining pin for roller, 12: cap for capsule body. 


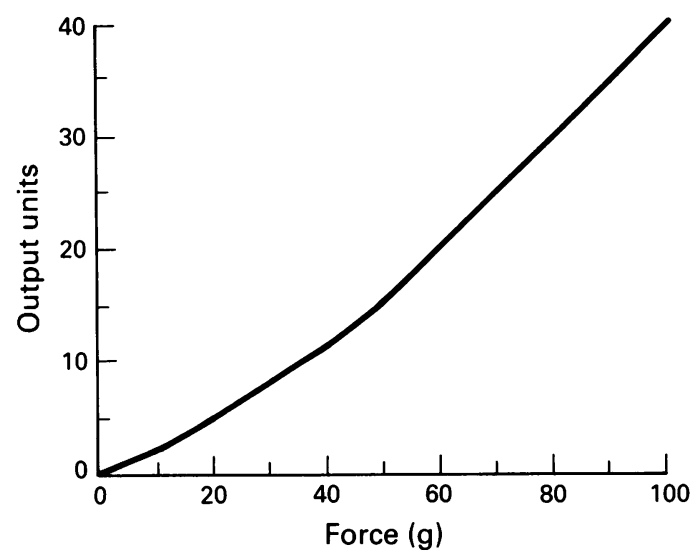

Figure 2: Graph of gram force versus output units created during calibration using 0-100 $\mathrm{g}$ weights.

\section{Methods}

\section{VOLUNTEERS}

After the application of topical anaesthesia to the pharynx 30 fasting normal asymptomatic volunteers (aged 24-38 years) swallowed the catheter assembly incorporating a force transducer and perfused side hole catheter system (Fig 1). The assembly was passed into the stomach and then withdrawn until the capsule was $5 \mathrm{~cm}$ above the lower oesophageal sphincter (recognised by a standard pull through technique). After a 10 minute rest period the response to $5 \times$ 'dry' swallows; $5 \times 5 \mathrm{ml}$ (water) and $5 \times 10 \mathrm{ml}$ (water) swallows was measured. In 10 of the subjects the response to $5 \times 15 \mathrm{ml}$ swallows was also assessed. There was a 30 second interval between swallows. The assembly was then resited with the capsule $10 \mathrm{~cm}$ above the lower oesophageal sphincter and the same sequence of swallows repeated after a five minute rest period. The sequence was repeated $15 \mathrm{~cm}$ above the lower oesophageal sphincter.

The study was approved by Research Advisory and Ethics Committee of Prince Henry's Hospital on 26 November 1985. All subjects gave informed consent before the commencement of the study.

FORCE TRANSDUCER ASSEMBLY (CAPSULE)

The force transducer is based on that described by Parrish et $a l^{5}$ and its construction is well described in Figure 1. The salient features are the saline filled silicone tubing looping around the pulley within the plastic capsule creating a force transducer with an effective length of $2 \mathrm{~cm}$. The stainless steel electrodes fitted into the two ends of the tubing are firmly fixed to the catheter assembly, such that the capsule can only be pushed away from the electrodes by stretching the silicone tubing and thus altering the resistance to the flow of any electrical current passed through the saline. A manometry channel is incorporated into the surface of the capsule and linked to the perfusion system (vide infra) by a loop of clear vinyl tubing so that distraction of the capsule from the catheter assembly is not impeded. The capsule assembly is $18 \mathrm{~mm}$ long with an outside diameter of $9 \mathrm{~mm}$

An alternate current impedance module measuring impedance values ranging from $0 \cdot 1-$ $150 \mathrm{Kohms}$ was connected to the electrodes. A stimulus current of 65 micro amps with a frequency of $6 \mathrm{khz}$ was used. Changes in impedance detected by the module were recorded on a Hewlett Packard recording system (paper speed $2.5 \mathrm{~mm} / \mathrm{sec}$ )

\section{CALIBRATION}

All calibrations were performed in a warm oven at $37^{\circ} \mathrm{C}$. Weights ranging from $1-200 \mathrm{~g}$ were suspended from the vertically supported force transducer and the impedance changes recorded to construct a calibration curve (Fig 2). The system had a response rate in excess of $200 \mathrm{~g} / \mathrm{sec}$. To test the effect of temperature on transducer function, calibration studies were performed in temperature environments ranging from $37^{\circ} \mathrm{C}$ to $4^{\circ} \mathrm{C}$. There was a slight difference in the output of the impedance monitor $(<10 \%$ variation) but only when the temperature went below $10^{\circ} \mathrm{C}$. In a separate study in one normal volunteer intraoesophageal temperature was recorded constantly for 30 minutes while $10 \mathrm{ml}$ boluses of tap water (temperature $20^{\circ} \mathrm{C}$ ) were ingested every 30 seconds. During this time intraoesophageal temperature ranged between 36.5 and $32 \cdot 1^{\circ} \mathrm{C}$. The lowest temperature was reached after eight minutes.

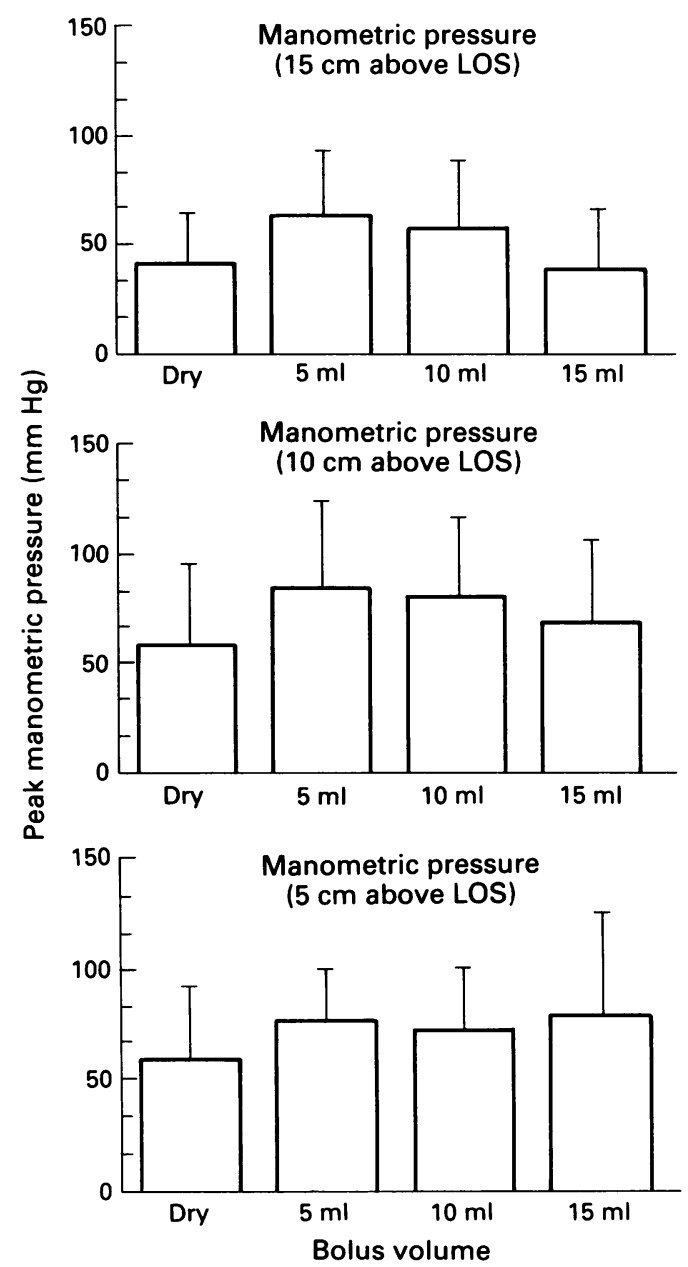

Figure 3: Histograms showing mean manometric pressure values $(\mathrm{mmHg})$ for each bolus volume at each site of measurement. 
MANOMETRY

Clear vinyl tubing (od $0.96 \mathrm{~mm}$, id $0.58 \mathrm{~mm}$ ) was bonded to form a six lumen catheter assembly to which the capsule (force transducer) was attached. Four channels were perfused with water $(0.6 \mathrm{ml} / \mathrm{min})$ by a pneumohydraulic system. ${ }^{6}$ Side holes were placed at 4,8 , and $12 \mathrm{~cm}$ above the capsule and changes in pressure were identified by Hewlett Packard quartz transducers and recorded on heat sensitive paper (at $25 \mathrm{~mm} / \mathrm{sec}$ ). The manometry system provided a response rate $>200 \mathrm{mmHg} / \mathrm{sec}$ with catheter compression.

\section{MEASUREMENTS AND ANALYSIS}

The following measurements were made: (a) 'Peak' force and pressure - defined as force rise (in grams) or pressure rise (in $\mathrm{mmHg}$ ) respectively from baseline to peak deflection; (b) duration - time in seconds from onset of rapid upstroke to end of downstroke (where it crossed baseline pressure) of force and pressure waves; (c) in the last 10 subjects studied all force and pressure recordings were stored directly onto a Macintosh SE computer through a Maclab digital to analogue converter. This allowed simple and accurate measurement of the areas under the force and pressure waves in these 10 subjects. The area was measured between the two points described for calculating duration. Only swallows which produced a peristaltic sequence, as assessed by review of the proximal recording sites, were analysed. If a double swallow was suspected it was not used for analysis and was repeated.

Mean and median values of peak force and peak pressure and duration were calculated for the five dry and five wet swallows $(5,10$, and 15 $\mathrm{ml})$ at each site $(5,10$, and $15 \mathrm{~cm}$ above the lower oesophageal sphincter). As the mean and median values were similar, only means are displayed in Figures 3 and 4 . The data were analysed using the Macintosh 'Statview' program supported by the super ANOVA (analysis of variance)

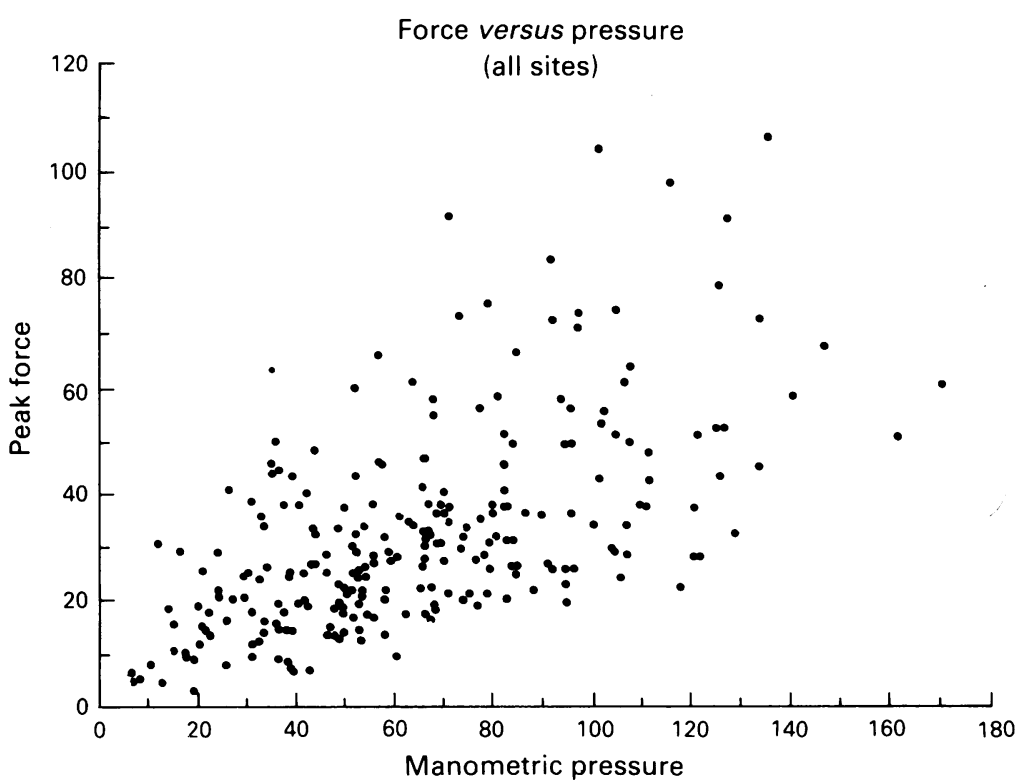

Figure 4: Scattergram of relationship between mean propulsive force and manometric pressure values at all sites and for all bolus volumes $(r=0 \cdot 61)$.
: File Edit Set-Up Speed Gain Display Analysis

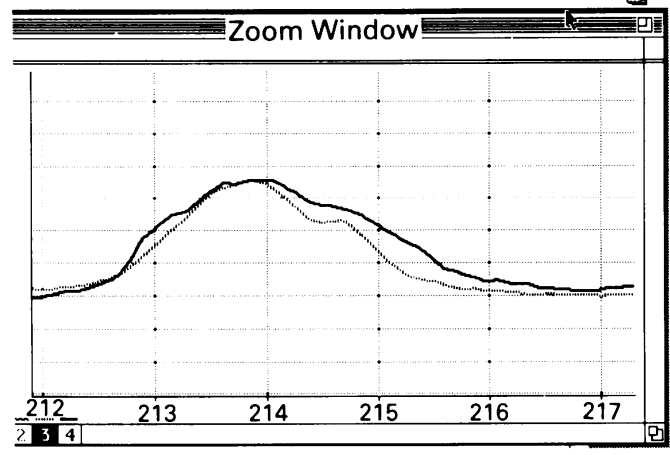

Channel 3: The manometric pressure wave recorded at the capsule

Channel 4: The propulsive force wave

Figure 5: Simultaneous propulsive force (solid line) and manometric pressure (broken line) recordings in response to a $10 \mathrm{ml} \mathrm{swallow}$ and measured at $10 \mathrm{~cm}$ above the lower oesophageal sphincter. This is the direct view from the computer screen obtained with the Mac lab program.

repeated measures analysis of variance software program in order to determine the influence of bolus size, and site within the oesophagus while allowing for the number of swallows performed by each individual subject. Differences between the means were compared using Schefe's method. This obviated the need to do stepwise multiple comparisons. The relationship between (a) peak propulsive force and peak manometric pressure and (b) duration of propulsive force and manometric pressure waves was assessed by Spearman's rank correlation coefficient.

\section{Results}

Figure 5 is a simultaneous tracing of propulsive force and manometric pressure waves recorded at $10 \mathrm{~cm}$ above the lower oesophageal sphincter and in response to $10 \mathrm{ml}$ bolus. The onset of the propulsive force and manometric pressure waves occurred simultaneously. Mean peak values of manometric pressure and propulsive force for each subject, generated in response to each bolus volume at the three measuring sites are shown in Figures 3 and 6 respectively.

\section{PROPULSIVE FORCE}

Effect of bolus volume (Fig 6)

The mean peak force rose progressively as bolus volume increased from dry to 5,10 , and $15 \mathrm{ml}$ at each site (analysis of variance, $F=13.6$, $p=0.0001)$. This rise was significant only for the difference between dry and wet swallows (dry $v 5 \mathrm{ml}, \mathrm{p}=0.0003$; dry $v 10 \mathrm{ml}, \mathrm{p}=0.0001$; dry $v 15 \mathrm{ml}, \mathrm{p}=0.002$ ). This rise was not seen between wet swallows as bolus volume increased from 5 to $15 \mathrm{ml}$.

\section{Effect of site (Fig 6)}

The mean peak force rose progressively as measurements were made more distally within the oesophagus (analysis of variance, $\mathrm{F}=12 \cdot 9$, $\mathrm{p}=0.0001)$. This rise was significant as the bolus 

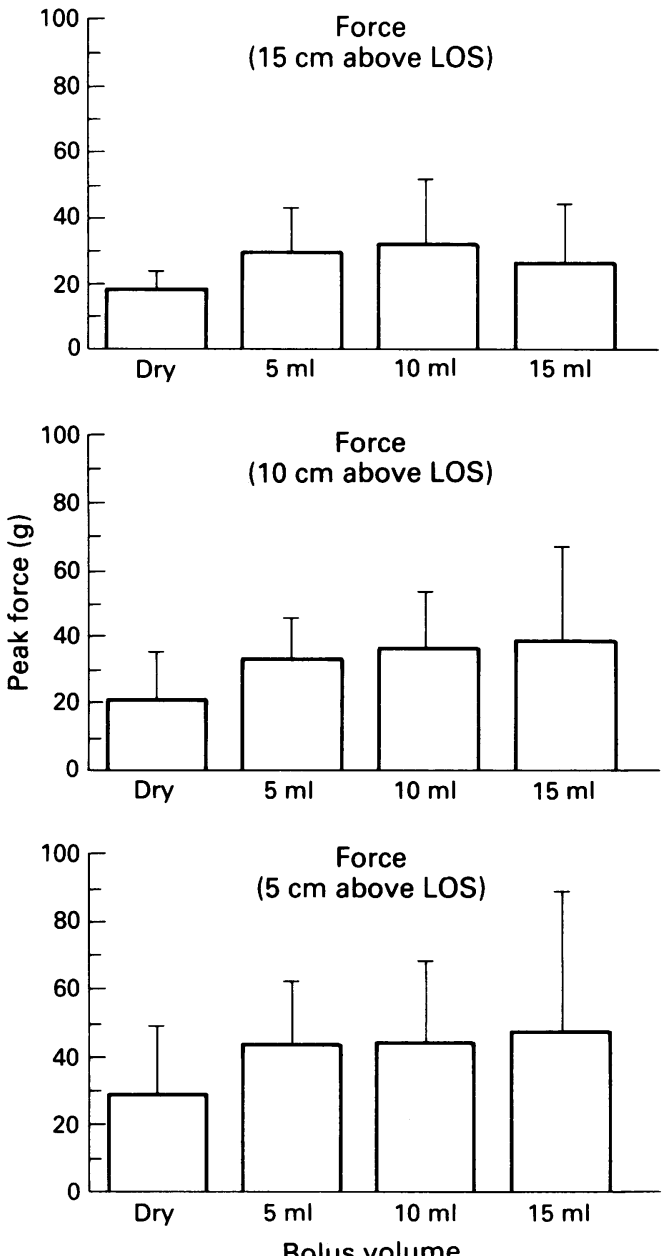

Figure 6: Histograms showing mean propulsive force values (g) for each bolus volume at each site of measurement.

moved from upper to lower oesophagus $(p=0.0001)$ or from mid to lower oesophagus $(\mathrm{p}=0.007)$.

\section{MANOMETRIC PRESSURE}

\section{Effect of bolus volume (Fig 3)}

The mean peak manometric pressure rose as bolus volume increased from dry to wet swallows (analysis of variance, $\mathrm{F}=7 \cdot 764, \mathrm{p}=0 \cdot 0001$ ). This rise was again not seen between wet swallows as bolus volume increased from 5 to $15 \mathrm{ml}$. This change was significantly different between dry

TABLE I Mean peak force values ( $g$ force) for each bolus volume at each level in the oesophagus

\begin{tabular}{lll}
\hline Site & $\begin{array}{l}\text { Bolus value } \\
(\mathrm{ml})\end{array}$ & $\begin{array}{l}\text { Peak propulsive force } \\
(\text { mean }(S D))\end{array}$ \\
\hline $5 \mathrm{~cm}$ above LOS & Dry & $28 \cdot 8(17 \cdot 6)$ \\
& 5 & $42 \cdot 4(16 \cdot 4)$ \\
& 10 & $43 \cdot 8(21 \cdot 8)$ \\
$10 \mathrm{~cm}$ above LOS & 15 & $47 \cdot 1(39 \cdot 3)$ \\
& Dry & $20 \cdot 8(12 \cdot 0)$ \\
& 5 & $33 \cdot 2(9 \cdot 8)$ \\
$15 \mathrm{~cm}$ above LOS & 10 & $36 \cdot 4(14 \cdot 8)$ \\
& 15 & $38 \cdot 7(25 \cdot 9)$ \\
& Dry & $17 \cdot 9(6 \cdot 5)$ \\
& 5 & $29 \cdot 3(11 \cdot 4)$ \\
& 10 & $31 \cdot 7(17 \cdot 1)$ \\
& 15 & $26 \cdot 0(15 \cdot 0)$ \\
\hline
\end{tabular}

LOS = lower oesophageal sphincter. and wet swallows only (dry $v 5 \mathrm{ml}, \mathrm{p}=0 \cdot 0004$; dry $v 10 \mathrm{ml}, \mathrm{p}=0 \cdot 003 ; \operatorname{dry} v 15 \mathrm{ml}, \mathrm{p}=0 \cdot 011$ ).

\section{Effect of site (Fig 3)}

The mean peak manometric pressure rose as the bolus moved down the oesophagus (analysis of variance. $F=12 \cdot 7, p=0 \cdot 0001)$. The changes were only significant between the upper and the two other sites, however, rather than between the mid and lower site (upper $v$ mid, $\mathrm{p}=0.0001$; upper $v$ lower, $\mathrm{p}=0 \cdot 004$ )

\section{Pressure/force relationship}

In general the pressure/force relationship showed low correlations at each site (Table I). The strongest correlation was seen with dry swallows at $10 \mathrm{~cm}$ above the lower oesophageal sphincter $(r=0 \cdot 69)$. Even when the pressure/ force correlation was examined separately in groups according to peak manometric pressure values $-<40 ; 40-100$, and $>100 \mathrm{mmHg}-$ no higher correlation was seen. The $40-100 \mathrm{mmHg}$ group encompassed approximately mean $+1 \mathrm{SD}$ and thus reflected the 'central' range of values. The overall correlation between pooled data was $\mathrm{r}=0.61$ (Fig 4).

By comparison, the overall correlation between the duration of the propulsive force and manometric pressure waves showed a higher correlation $(\mathrm{r}=0.79)$ (Table II) and was higher for wet than dry swallows.

The overall correlation between the areas under the manometric pressure and propulsive force waves was also low $(r=0.59)$ (Table III) and was higher for dry then wet swallows in the subjects examined $(n=10)$.

\section{Discussion}

We have studied the oesophageal propulsive force and its relationship to the manometric pressure wave. Our results show that the propulsive force and manometric pressure waves have a simultaneous onset and that the duration of both waves is generally similar. Peak propulsive force values increased as we measured progressively more distally within the oesophagus and as we changed from dry to wet swallows. Only a weak relationship between peak propulsive force and peak manometric pressure values was noted along the length of the oesophagus. The highest correlation was seen with dry swallows at $10 \mathrm{~cm}$ above the lower oesophageal sphincter.

It may be hazardous to compare the results of this study with those of the other two studies ${ }^{2}$ because of differences in methodology. We have confirmed that propulsive force is greatest in the distal oesophagus, but differ from Pope ${ }^{2}$ in that wet swallows produced significantly greater peak force values than dry swallows. We agree with Pope $^{2}$ that in general the relationship between peak propulsive force and manometric pressure values is weak. Differences in methodology most probably account for these conflicting results. Our force transducer was similar to that described by Parrish et al and used by Pope and Schoen,,$^{3}$ but we have substituted saline for mercury as the conducting medium for the 
TABLE II Mean peak manometric pressure values $(\mathrm{mmHg})$ for each bolus volume at each level in the oesophagus

\begin{tabular}{lll}
\hline Site & $\begin{array}{l}\text { Bolus value } \\
(\mathrm{ml})\end{array}$ & $\begin{array}{l}\text { Peak manometric } \\
\text { pressure }(\text { mean }(S D))\end{array}$ \\
\hline $5 \mathrm{~cm}$ above LOS & Dry & $59 \cdot 0(29 \cdot 2)$ \\
& 5 & $76 \cdot 5(20 \cdot 1)$ \\
& 10 & $72 \cdot 0(26 \cdot 2)$ \\
$10 \mathrm{~cm}$ above LOS & 15 & $78 \cdot 0(44 \cdot 1)$ \\
& Dry & $58 \cdot 1(33 \cdot 2)$ \\
& 5 & $83 \cdot 6(37 \cdot 1)$ \\
$15 \mathrm{~cm}$ above LOS & 10 & $80 \cdot 0(32 \cdot 7)$ \\
& 15 & $68 \cdot 3(34 \cdot 6)$ \\
& Dry & $40 \cdot 7(20 \cdot 0)$ \\
& 5 & $62 \cdot 4(27 \cdot 3)$ \\
& 10 & $56 \cdot 9(27 \cdot 5)$ \\
& 15 & $38 \cdot 1(23 \cdot 7)$ \\
\hline
\end{tabular}

following reasons. Saline is less sensitive to temperature changes, although we did find that force values measured in vitro did vary with temperature. This variation was sufficient to require us to perform all subsequent calibrations at $37^{\circ} \mathrm{C}$ and to question Schoen's finding: 'Temperature did not change the calibration curves for each gauge'. Saline is safer than mercury in the event of mechanical disruption causing spillage into the gut. The ideal stimulus current necessary for a saline system is much less than for a mercury system (factor of 1000) and is well within safe physiological limits. We believe our design and construction, like previous systems ${ }^{23}$ ensure that the capsule can only be distracted from the catheter assembly by stretching the silicone tubing (force transducer). We accept that despite anchoring the catheter assembly to the nose, some movement will occur, but have assumed this will not prejudice our measurement of peak propulsive force. In an attempt to minimise any inaccuracy in manometric values we incorporated a manometry channel into the surface of the capsule and used a modern low compliance perfusion system. ${ }^{6} \mathrm{We}$ did, however, find that peak manometric pressure recorded at the capsule surface was similar to that recorded from one of the measuring ports on the catheter when that port was recording from the same level on repositioning the catheter.

Our study examines more closely the relationship between the propulsive force and manometric pressure waves. The simultaneous onset and the similar duration of both waves does confirm the obvious hypothesis that the manometric pressure wave is indeed responsible for the propulsive force wave we have measured. The poor relationship between peak propulsive force and peak manometric pressure values indicates that the peak amplitude of the manometric pressure wave as analysed in this study was a poor predictor of propulsive force. Even when the integrated response to deglutition (area under manometric pressure and propulsive force

TABLE III Relationship between areas under manometric pressure and propulsive force waves in 10 subjects

\begin{tabular}{llll}
\hline & \multicolumn{4}{l}{ Correlation coefficients (on means) } \\
\cline { 2 - 4 } & \multicolumn{4}{l}{ Distance above LOS } \\
\cline { 2 - 4 } Bolus volume & $5 \mathrm{~cm}$ & $10 \mathrm{~cm}$ & $15 \mathrm{~cm}$ \\
\hline Dry & $\mathrm{r}=0.48$ & $\mathrm{r}=0.69$ & $\mathrm{r}=0.84$ \\
Wet & $\mathrm{r}=0.17$ & $\mathrm{r}=0.68$ & $\mathrm{r}=0.37$ \\
\hline
\end{tabular}

waves) was compared, the manometric pressure wave failed to predict the impulse (area under propulsive force wave (force $\times$ time)) developed in response to deglutition. This is further evidence that the manometric (intraluminal) pressure changes measured at manometry are not an absolute measure of oesophageal function. This supports the elaborate study by Kahrilas et $a l^{7}$ showing that normal bolus transit occurred within a wide range of manometric pressure values. Kahrilas et al, however, did find that a critical manometric pressure amplitude $(>30$ $\mathrm{mmHg}$ ) was necessary for normal transit in the distal oesophagus. They also showed that distal movement of the tail of the bolus coincided with the onset of the upstroke of the manometric pressure wave. Before, further comparisons are made the 'origin' of the propulsive force wave and any factors that may alter it must be considered. As a wave of peristaltic contraction passes down the oesophagus it obliterates the lumen (to a varying degree) and the capsule comprising the force transducer is gripped and displaced in an aboral direction. The initial aboral displacement will remove any inherent laxity in the catheter assembly and its fixation, but any further displacement must result from stretching of the saline filled silicone tubing of the force transducer (Fig 1). Displacement will then occur till the degree of stretch equivalent to the maximal downward force (peak propulsive force) has been attained then no further displacement is possible and the peristaltic wave must simply slide distally over the capsule and release it from its grip. The factors that will affect maximal downward force can be resolved into two practical components - the degree of 'grip' exerted by the occluding wave as it passes distally and the ability of the oesophageal musculature to direct the wave distally against a fixed intra luminal object. The degree of grip on the capsule could be altered in a number of ways: (a) capsule diameter, (b) degree of luminal obliteration caused by the peristaltic wave, and (c) the friction between mucosa and capsule surface. The previous studies did show that peak propulsive force increased with capsule diameter ' : but decreased when friction was reduced by ingestion of salad oil.' We have left friction and capsule diameter constant in this present study of the effect of site of reassurement of bolus volume on propulsive force and its relationship with the manometric pressure wave.

When the passage of a liquid barium bolus, ingested in the supine position, is observed fluoroscopically, it is seen to spread out along the length of the oesophagus as a relatively narrow column of barium. As the column passes down the oesophagus it shortens and becomes more compact in the distal oesophagus (phase 1 of transit) before passing distally into the stomach (phase 2 of transit). Our own studies with nonviscous liquid radionuclide boluses confirm the barium studies and show that in the supine position phase 1 of transit occurs under the action of the pharynx and before the appearance of the peristaltic wave in the proximal oesophagus. ${ }^{\text {T }}$ The oesophagus can be so efficient as a simple conduit that on occasions even in the supine position non-viscous liquid boluses may 
enter the stomach before the onset of the peristaltic waves. ${ }^{8}$ The studies of Sugarbaker et $a l^{9}$ in the opossum have shown that longitudinal muscle contraction preceeds the circular group and that this contraction of the longitudinal muscle is of longer duration. These authors have suggested this may provide advancing rigidity to the conduit (oesophagus). ${ }^{9}$

Phase 2 of bolus transit generally requires the oesophageal peristaltic wave. Before peristaltic action sweeping the bolus distally into the stomach the bolus has become more compact requiring the distal oesophagus to accommodate a greater volume per unit area. This may cause distension and activation of stretch receptors whose presence has been postulated by others. ${ }^{10}$ In vitro studies have shown a rise in the force of contraction of oesophageal muscle in response to increasing the stretch. ${ }^{112}$ This might account for the distal rise in peak propulsive force values that is, the greater the volume and hence the preload on the oesophageal muscle then the greater the force generated. In our study, although peak manometric pressure rises as the wave moves from $15 \mathrm{~cm}$ to $10 \mathrm{~cm}$ above the lower oesophageal sphincter there is no further stepwise increase as one measures more distally within the oesophagus or as bolus volume increases. This lack of rise may be real, however, as we measured pressure at only three sites and as there is a large variance in individual manometric data, a true rise in pressure may be obscured.

In conclusion, we have compared certain features of the oesophageal propulsive force and manometric pressure waves. The temporal relationship between the waves indicates that they are both part of the same basic mechanism. The weak correlation between propulsive force and manometric pressure values would suggest that manometric pressure is a poor predictor of propulsive force and may be a poor measure of oesophageal function. Further information on the pressure/force relationship may be gained by altering the manometric pressure wave with pharmacological agents with a known effect - for example, atropine, bethanecol.

1 Russell C, Whelan G. Oesophageal manometry: how well does it predict oesophageal function. Gut 1987; 28: 940-5.

2 Pope CE, Horten PF. Intraluminal force transducer measurements of human oesophageal peristalsis. Gut 1972; 13: 464 70 .

3 Schoen HJ, Morris DW, Cohen S. Oesophageal peristaltic force in man. Dig Dis 1977; 22: 589-97.

4 Arndorfer R, Stef J, Dodds W, et al. Improved infusion system for intraluminal oesophageal manometry. Gastroenterology 1977; 73: 23-7.

5 Parrish D, Strandness DE Jnr, Bell JW. Dynamic response characteristics of a mercury in silastic strain gauge. $\mathcal{F} A p p l$ Physiol 1964; 19: 363-5.

6 Dent J, Culross J, Morris J. A pneumatically driven pump for constant perfusion manometry. Aust $\mathcal{f}$ Exp Biol Med Sci constant perfusion manometry. Aust $\mathcal{F}$ Exp Biol

7 Kahrilas PJ, Dodds WJ, Hogan WJ. Effect of peristaltic dysfunction on esophageal volume clearance. Gastroenterology 1988; 94: 73-80.

8 Buthpitya G, Stroud D, Russell C. Pharyngeal Pump and Oesophageal Transit. Dig Dis Sci 1987; 32: $1244-9$.

9 Sugarbaker D, Rattan S, Goyal R. Swallowing induces sequential activation of oesophageal longitudinal smooth muscle. Am 7 Physiol 1984; 247: G515-9.

10 Assad K, Rahman S, Nawar N, Milkhaily. Intrinsic innervation of the oesophagus in dogs with special reference to the presence of muscle spindles. Acta Anat 1983; 115: 91-6.

11 Cohen S, Green F. Mechanics of oesophageal muscle contraction. Evidence of an inotropic effect of gastrin. $\mathcal{F}$ Clin Invest tion. Evidence

12 Cohen S, Green F. Force velocity characteristic of oesophageal muscle. Effect of acetylcholine and norepinephrine. Am F Physiol 1973; 226: 1250. 